\title{
Computer modeling studies of the structural role of NADPH binding to active site mutants of human dihydrofolate reductase in complex with piritrexim ${ }^{\star \star}$
}

\author{
Wiesław Nowak ${ }^{1}$, Vivian Cody $^{2}$ and Andrzej Wojtczak ${ }^{3}$ \\ ${ }^{1}$ Institute of Physics, Nicholas Copernicus University, Toruń, Poland; ${ }^{2}$ Hauptman-Woodward \\ Medical Research Institute, Buffalo, U.S.A.; ${ }^{3}$ Institute of Chemistry, Nicholas Copernicus \\ University, Toruń, Poland
}

Received: 19 November, 2001; accepted: 3 December, 2001

Key words: chemotherapy, enzyme structure, dihydrofolate reductase, piritrexim, NADPH, computer modeling, ESFF forcefield

\begin{abstract}
Dihydrofolate reductase (DHFR, EC 1.5.1.3) is one of the enzymes active in the folate cycle which plays an important role in DNA synthesis. Inhibition of DHFR is a key element in the treatment of many diseases, including cancer and AIDS related infections. A search for new selective inhibitors is motivated by the resistance to common drugs observed in the course of treatment. In this paper, results of a detailed computer analysis of human DHFR interactions with the lipophilic inhibitor piritrexim (PTX) are presented. It was found that the NADPH cofactor contributes $30 \%$ of the total PTX-enzyme interaction energy. Substitution of the highly conserved Glu30 with alanine does not lead to the release of the inhibitor from the hDHFR pocket. The important L22F point mutation does affect PTX orientation but does not change the binding energy. Simulations of the dynamics of binary hDHFR-PTX complexes were performed with the use of Extensible Systematic Force Field (ESFF) and the results indicate structural changes in the enzyme induced by NADPH binding.
\end{abstract}

\footnotetext{
${ }^{\star}$ Presented at the XXXVII Convention of the Polish Biochemical Society, Torun, Poland, September, 10-14, 2001.

This work was supported by grant UMK 414-F (WN) and in part by FIRCA Fogarty R03 TW 00789 (VC). The authors thank the State Committee for Scientific Research (KBN, Poland) for partial support of the nation wide license of the MSI (currently Accelrys) software.

${ }^{\star}$ To whom correspondence should be addressed: Wiesław Nowak, Institute of Physics, Nicholas Copernicus University, Grudziądzka 5, 87-100 Toruń, Poland; phone: (48 56) 611 3204;

e-mail: wiesiek@phys.uni.torun.pl
}

Abbreviations: hDHFR, human dihydrofolate reductase (5,6,7,8-tetrahydrofolate:NADP oxidoreductase, EC1.5.1.3); ecDHFR, Escherichia coli dihydrofolate reductase; NADPH, nicotinamide adenine dinucleotide phosphate, PTX, piritrexim, 2,4-diamino-6-(2,5-dimethoxybenzyl-5-methylpyrido[2,3-d]pyrimidine; TMP, trimethoprim, 2,4-diamino-5-(3,4,5-trimethoxybenzyl)-pyrimidine, MTX, methotrexate, 4-amino-4-deoxy-10-methylfolic acid; ESFF, Extensible Systematic Force Field. 
Dihydrofolate reductase (DHFR, EC1.5.1.3, Fig. 1) catalyzes the reduction of 7,8-dihydro-

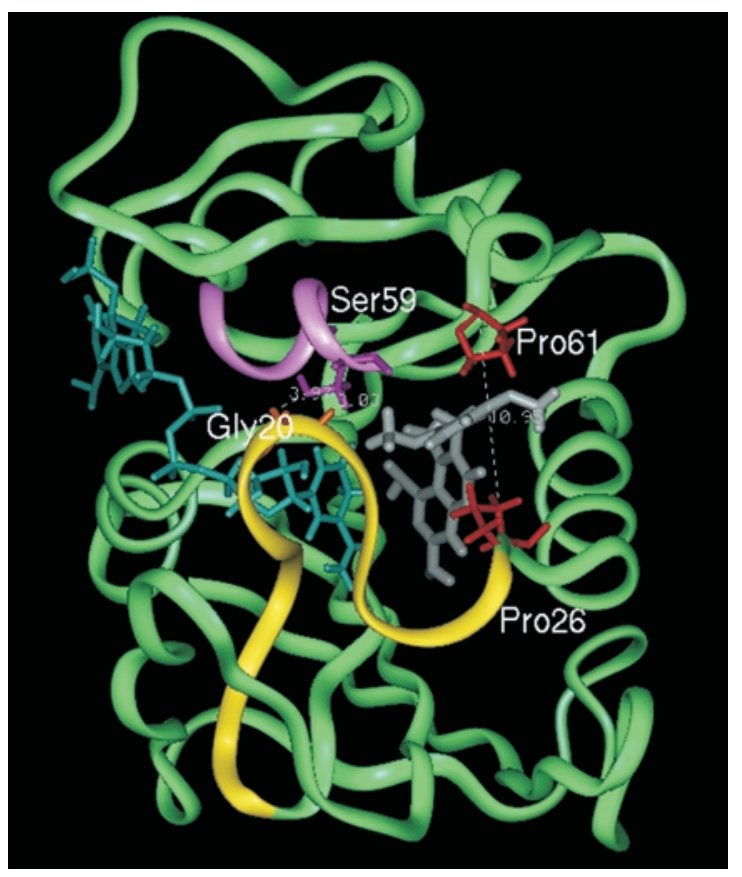

Figure 1. Secondary structure of human dihydrofolate reductase.

The position of the piritrexim inhibitor (grey) and NADPH cofactor (blue) are shown in their binding sites. Loop I and helix C, involved in the cofactor binding, are colored yellow and magenta, respectively. Side chains of residues discussed in the text are displayed in stick representation (red).

folate to 5,6,7,8-tetrahydrofolate, in which NADPH is a coenzyme [1]. Tetrahydrofolate is the source of methyl groups in the synthesis of purines and pyrimidines, as well as some amino acids. Inhibition of DHFR activity leads to cell death, therefore this enzyme has been extensively studied as a drug target for bacterial, protozoal and fungal infections. It is also an important target of chemotherapy in neoplastic and autoimmune diseases. Examples of the widely used antifolates are the potent antibacterial agent trimethoprim (TMP) and the antineoplastic drug methotrexate (MTX) [2]. Recent studies have shown [3] that mutants of hDHFR, in particular F31X [4] and L22X [5], have increased resistance to the cytotoxic effects of classical antifolates. Piritrexim (PTX, 2,4-diamino-6-(2,5-dimetho- xybenzyl)-5-methylpyrido[2,3d]-pyrimidine) (Fig. 2) was developed as a lipophilic

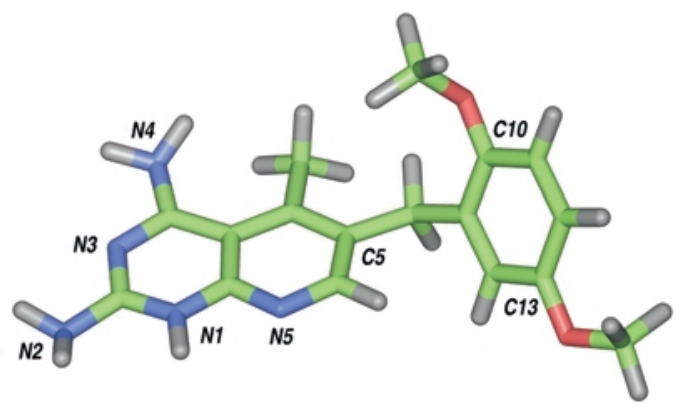

Figure 2. The structure of piritrexim (PTX).

antifolate [6] to overcome problems related to the specific folate transport system required in mammalian cells by classical drugs.

PTX is a better inhibitor for the L22F mutant of hDHFR found in some MTX-resistant tumors, than the classical anifolates [5] and phase II clinical trials show its efficacy in the treatment of cancer of the bladder [7]. In a set of 20 engineered hDHFR variants tested, PTX had better cytotoxic properties than the more traditional drugs [3]. Additional interest in PTX and related compounds was further motivated by its promise in the treatment of AIDS-related infections ([8] and references therein). It is worth noting that PTX is able to inhibit DHFR from Kaposi's sarcoma-associated herpes virus with $K(\mathrm{i})=3.9 \mathrm{nM}$ [9].

To understand the binding interactions of PTX, in particular with the MTX-resistant variants of hDHFR, the crystal structures of the ternary hDHFR-NADPH-PTX complexes were investigated $[5,10]$. These structures revealed that the conformation of PTX was variable depending on the nature of the active site mutant and whether the structure was the ternary or the binary complex. These data suggest that the conformational flexibility of PTX may be an advantage in overcoming the MTX-resistance observed in these mutants. The structure of the wild type hDHFR ternary complex with PTX and NADPH was the basis of previous theoretical studies carried out in our laboratory [11], which showed a general 
agreement with the observed crystal structure results.

Bacterial ecDHFR complexes have been studied with respect to the large scale conformational changes related to its catalytic activity [12, 13]. NMR and crystal structure data have shown that both the flexible M20 loop and the $\beta \mathrm{F}-\beta \mathrm{G}$ loop play an important role in controlling access to the active site of ecDHFR [14]. NMR data for hDHFR ternary complexes of PT523 and NADPH [15] suggest that such conformational flexibility may also exist in the human DHFR structure. While crystal structure data for the PT523 ternary complex [16] showed that the ligand binds with multiple conformations, there was no indication that the enzyme exhibited ligand-induced conformational flexibility. To further characterize the conformational properties of hDHFR, molecular dynamics (MD) studies of loop motions in the human enzyme were carried out for various active site mutants, and the results are presented in this paper.

Another aspect of this study stems from the recent general hypothesis of Pan et al. [17] that the allosterism and signal transduction of DHFR may be a result of changes in the distribution of states within the ensemble of the enzyme. An analysis of ecDHFR shows that, in addition to pair-wise coupling of residues, there exists a functional connectivity between binding sites. We believe that MD simulations and analysis of the computed interaction energies presented here provide useful insights into such general problems.

In particular, we address the question of to what extent the position of PTX is affected by NADPH binding. Substrate-coenzyme cooperativity in bacterial ecDHFR systems has been characterized [18] but this issue has not been adequately addressed in the bigger and more rigid human enzyme. In general the NADPH binding pocket properties determine the selectivity of the enzyme towards this particular cofactor and the pocket architecture is the main factor which discriminates between NADP and NADPH binding. Thus the coenzyme pocket structure is critical for the type of the enzymatic activity performed by the protein [19]. In certain cases strong $\mathrm{NADPH}$-inhibitor interactions may even result in the displacement of the dihydronicotinamide moiety from the binding pocket of DHFR [20]. Thus, our MD simulations were aimed at a structural comparison of the binary hDHFR-PTX and the ternary hDHFRNADPH-PTX complexes.

The computational ESFF method is novel for protein studies. Computer modeling of DHFR enzymes have been the focus of several research groups [21-26] but our approach has the advantage of providing a tool for fast and efficient study of any inhibitor, without the necessity for the difficult and time consuming phase of forcefield parameter development.

\section{METHODS}

Computer simulation results depend on the quality of the forcefield. The ESFF forcefield [27], as implemented in the InsightII v. 97.0 package [28], was chosen for its ability to recalculate on-the-fly the partial charges based on the local environment of the atoms. This feature is particularly important in our case, since NADPH carries a substantial charge, and changes in the electrostatics are easily accommodated within this model. ESFF is a rule-based forcefield covering almost all atoms in the periodic table. For each atom type, electronegativity and hardness obtained from density functional theory (DFT) calculations, together with atomic radii, are kept in a special library. These quantities are converted into forcefield parameters using a set of rules. The parametrization accounts for such features as bond order, ring size, whether bonds are endo or exo to rings and, for metal ligands, the cis-trans and axial-equatorial positioning. It is a diagonal forcefield, with Morse bond potentials. The charges are determined in a complex way, by minimizing the electrostatic energy under the constraints that the 
sum of the charges is equal to the net charge of the molecule. One should note that van der Waals parameters are affected by the charge of an atom. An important part of modeling is correctly setting-up all bond orders in the system under study.

ESFF gives excellent results for structures of small complexes and is one of a few forcefields good for organometallics. An advantage of using the ESFF forcefield is the ease in automatic parameterization of all atom types, which gives good prospects for massive screening for new inhibitors of DHFR or other drug design targets.

The X-ray crystal structure of wild type DHFR-NADPH-PTX complex [10] refined to $2.3 \AA$ was used as a starting point for further modeling. All 100 crystallographic water molecules were retained in the model. Hydrogen atoms were added using the "Biopolymer" module of the InsightII package. The same module was used to substitute the leucine in position 22 with phenylalanine (L22F) and the glutamic acid in position 30 with alanine (E30A). The "soak" option of "Biopolymer" was used to generate a $6 \AA$ layer of water molecules (1693 water molecules) resulting in over 7500-atom models of the complexes. The PTX ligand was protonated at the $\mathrm{N} 1$ atom (net charge +1 ) and NADPH had net charge of -3 . The only amino acids charged in the model (Glu30, Lys54, Lys55, Arg77) gave the zero total charge of the investigated ternary systems.

The group-based method was used in the non-bonded interactions energy evaluation. Both the wt and the L22F hDHFR models of the ternary complexes were subjected to 200 steps of the conjugate gradient method (PolakRibiere) minimization (module "Discover3" of InsightII v. 97.0), followed by 20 steps of water shell optimization with the protein part held fixed. At this minimization stage, a large $30 \AA$ non-bonded interactions cutoff with $1 \AA$ wide buffer switching region was used. The ESFF minimized structures compare well with the X-ray structures [11].
The initial model of the binary complex was obtained from the ESFF minimized wt hDHFR ternary complex by replacing all heavy atoms of NADPH by randomly oriented water molecules (W2). The total charge of $+2 \mathrm{e}$ for the binary complex was used with the negative charge on Asp21. This modification was introduced in the carboxylic residue near the active site that has the shortest distance to Leu22. The negative charge of Asp21 mimics to some extent the effect of the negatively charged NADPH coenzyme, which is absent in the binary complex, thus such an approach brings the total charge of the system closer to neutrality. In the initial model of the binary complex the protein structure was held rigid for 200 cycles of ESFF minimization, then the whole complex was minimized for additional 200 cycles. Molecular dynamics were run for $10 \mathrm{ps}$ for this system at $298 \mathrm{~K}$. The last structure was used to make the E30A mutation (module "Biopolymer"). The E30A variant was subjected to 3 ps equilibration and after 200 cycles of minimization the structure was used in further analysis.

In order to reduce the computational time, PTX and NADPH were divided into small charge groups and subsequent calculations were thus done with $15 \AA$ cutoffs. A dielectric constant of 1 was used through all computations. All bond lengths were kept constant using the RATTLE algorithm [29]. The simulation timestep was $1 \mathrm{fs}$. The initial temperature was $10 \mathrm{~K}$. During the dynamics simulations the temperature was kept constant at $298 \mathrm{~K}$ by velocity scaling. Typical heating $(<1000$ steps) and equilibration phases were short. Selected geometrical parameters and total energies showed stablization starting from 5-6 ps. The rms for $\mathrm{C} \alpha$ atoms of the binary complex has stablized at the level of $1.35 \AA$ after 8 ps. The data were collected each 0.1 ps through 10-30 ps and analyzed using the InsightII 97.0 and PROCHECK [30] tools. Calculations were performed on an SGI $\mathrm{O} 2$ R5000 workstation. 


\section{RESULTS AND DISCUSSION}

Our previous study showed that the strongest interactions of PTX are those formed with NADPH and Glu30 [11]. Therefore, one may expect that these particular forces play a major role in inhibitor docking. The experimental ometries and protein structures taken as snapshots from MD trajectories. The comparison showed that about $75 \%$ of the amino acids in the models subjected to MD are found in the most favored regions of the Ramachandran plot, while in the crystal structure almost $92 \%$ residues were found in these regions (Ta-

Table 1. Ramachandran data (\%) for $\phi / \psi$ angles of ternary (3) and binary (2) complexes of DHFR. The symbol MIN denotes minimized structures, $10 \mathrm{ps}$ and $30 \mathrm{ps}$ indicate the corresponding snapshots from the MD trajectories.

\begin{tabular}{lccccc}
\hline Region & (3) wt & (3) wt & (3) L22F & \multicolumn{1}{c}{$(2)$} & \multicolumn{1}{c}{$(2)$} \\
& X-ray & MIN & $30 \mathrm{ps}$ & MIN & $10 \mathrm{ps}$ \\
\hline Most favored & 91.8 & 84.3 & 76.2 & 75.5 & 72.3 \\
Allowed & 8.2 & 14.5 & 22.6 & 22.6 & 26.4 \\
Generously allowed & - & 1.2 & 1.2 & 1.9 & 1.3 \\
\hline
\end{tabular}

structures of the binary PTX complexes of wt hDHFR and the E30A variant are not known so computer models of these systems were generated as described in the Methods section. Properties of the binary complexes are analyzed here and compared with ternary systems in order to determine the role of NADPH in PTX binding to the enzyme.

\section{Modeling of hDHFR-PTX binary complexes}

The starting crystal structure was refined with the X-PLOR program [31] and CHARMM forcefield to the resolution of $2.3 \AA$ [10]. The models of the binary complexes and the ternary L22F variant complex were compared to the crystal structure. The overall conformation of the polypeptide chain of hDHFR was maintained during the minimization and simulations. The rms differences between the minimized models and the crystal structure of the ternary complex [10], calculated for the $\mathrm{C} \alpha$ positions, are 1.05, 1.06 and $0.98 \AA$ for the wt hDHFR-PTX, E30A-PTX and L22F-NADPH-PTX complexes, respectively.

The PROCHECK program [30] was used to assess the overall quality of the minimized ge- ble 1). Although the ESFF minimization gave $1-2 \%$ of residues in the generously allowed regions, which were not present in the crystal structure, the ESFF polypeptide conformation should be considered good.

The common feature of all the ESFF structures was the loss of planarity for the peptide bonds (Table 2) described by the omega torsion angle. The estimated standard deviation for this value in the typical crystal structures, at $2.3 \AA$ resolution, is $6.0^{\circ} \pm 3.0^{\circ}$. In the initial $\mathrm{X}$-ray structure it was $2.2^{\circ}$, while for the minimized system it was up to $9.8^{\circ}$ and for the MD frame $12.2^{\circ}$.

The side chain conformations for all the residues in the ESFF minimized structures, monitored in terms of $\chi 1 / \chi 2$ pairs of torsion angles, are closer to the idealized geometries than those from the X-ray analysis (Table 3).

The analysis performed shows that the moderate ESFF minimization of the DHFR protein ternary and binary complexes leads to realistic structures. The planarity of the peptide bonds may be, however, of some concern in ESFF protein models, and should be monitored in future simulations. 
Table 2. Main chain parameters for the main chain atoms of the crystal structure and ESFF complexes of wt hDHFR, the L22F variant and the binary complex.

For symbols used see Table 1.

\begin{tabular}{|c|c|c|c|c|c|c|c|}
\hline Stereochemical Parameter & $\begin{array}{l}\text { (3) wt } \\
\text { X-ray }\end{array}$ & Typical $^{\text {b) }}$ & $\begin{array}{l}\text { (3) } \\
\text { MIN }^{\text {a) }}\end{array}$ & $\begin{array}{l}\text { (3) L22F } \\
\text { MIN }^{\text {a) }}\end{array}$ & $\begin{array}{l}\mathrm{L} 22 \mathrm{~F} \\
30 \mathrm{ps}\end{array}$ & $\begin{array}{l}\text { (2) } \\
\text { MIN }\end{array}$ & $\begin{array}{l}(2) \\
10 \mathrm{ps}\end{array}$ \\
\hline$\%$ Most favored & 91.8 & $82.6 \pm 10.0$ & 84.3 & 80.5 & 76.2 & 75.5 & 72.3 \\
\hline Omega angle & 2.2 & $6.0 \pm 3.0$ & 9.2 & 9.1 & 9.1 & 9.8 & 12.2 \\
\hline Bad contacts $/ 100$ res. & 1.1 & $5.2 \pm 10.0$ & 0.5 & 0.5 & 0.0 & 0.0 & 0.5 \\
\hline Zeta angle & 1.8 & $3.1 \pm 1.6$ & 2.0 & 1.9 & 1.9 & 2.0 & 3.9 \\
\hline H-bond E & 0.8 & $0.8 \pm 0.2$ & 0.8 & 0.7 & 0.8 & 0.8 & 0.8 \\
\hline
\end{tabular}

a) from [11]; b) Typical value for structures at $2.3 \AA$ resolution; mean \pm st. dev.

Table 3. Standard deviations of the side chain torsion angles for the crystal structure and the modeled complexes of hDHFR.

For symbols used see Table 1.

\begin{tabular}{|c|c|c|c|c|c|c|c|}
\hline Torsion angle & $\begin{array}{l}\text { (3) wt }{ }^{a)} \\
\text { X-ray }\end{array}$ & Type $^{\text {b) }}$ & (3) $\mathrm{MIN}^{\mathrm{a})}$ & $\begin{array}{l}\text { (3) } \mathrm{L} 22 \mathrm{~F} \\
\mathrm{MIN}^{\text {a) }}\end{array}$ & $\begin{array}{l}\mathrm{L} 22 \mathrm{~F} \\
30 \mathrm{ps}\end{array}$ & $\begin{array}{l}(2) \\
\text { MIN }\end{array}$ & $\begin{array}{c}(2) \\
10 \mathrm{ps}\end{array}$ \\
\hline$\chi_{1}$ gauche(-) & 17.2 & $19.0 \pm 6.5$ & 8.2 & 7.6 & 8.5 & 8.4 & 13.2 \\
\hline$\chi_{1}$ trans & 14.0 & $19.7 \pm 5.3$ & 16.0 & 15.5 & 9.8 & 14.4 & 11.8 \\
\hline$\chi_{1} \operatorname{gauche}(+)$ & 13.8 & $18.3 \pm 4.9$ & 9.8 & 10.8 & 9.1 & 9.5 & 12.9 \\
\hline$\chi_{1}$ pooled & 14.7 & $18.9 \pm 4.8$ & 13.0 & 13.0 & 10.2 & 12.3 & 13.4 \\
\hline$\chi_{2}$ trans & 14.2 & $20.9 \pm 5.0$ & 10.5 & 11.6 & 1.4 & 13.2 & 15.2 \\
\hline
\end{tabular}

a) from [11]; b) Typical value for structures at $2.3 \AA$ resolution; mean \pm st. dev.

\section{NADPH effect on hDHFR-PTX complex structure}

Repetitive NADPH cofactor binding and dissociation is a basic process in the DHFR catalytic cycle [12]. The presence of NADPH in the DHFR cleft may lead to either positive or negative cooperative effects of ligand binding, depending on the type of inhibitor and/or the structure of the protein. Such a situation is observed, for example, in MTX complexes of ecDHFR variants [5]. Molecular dynamics simulations of Lactobacillus casei DHFR revealed a long range correlation between the adenosine fragment of NADPH and the p-aminobenzoyl fragment of MTX [21] which may contribute to the positive cooperativity in this system. The role of NADPH in the binding of lipophilic inhibitors (PTX) has not been studied so far. Using the Discover3 software it was possible to calculate interaction energies between selected parts of hDHFR and PTX.

Data presented in Table 4 (ternary complexes) and Table 5 (binary systems) show that positive NADPH cooperativity with respect to PTX binding should be expected. This statement is based on the large value of -33 $\mathrm{kcal} / \mathrm{mol}$ for the NADPH-PTX interaction energy found both in the wt and L22F variants of hDHFR (see Table 4). The strongest stabilization of the inhibitor $(-65 \mathrm{kcal} / \mathrm{mol})$ comes from Glu30. Contributions from other residues to PTX binding energy are much smaller. The main residues - Val115, Arg28 and Arg32 - give only about $-5 \mathrm{kcal} / \mathrm{mol}$ each. 
Table 4. Piritrexim (PTX) intermolecular interaction energy (kcal/mol) calculated for wt hDHFR and L22F hDHFR ternary complexes using the ESFF forcefield in Discover3.

The calculated energy is partitioned between the selected amino acids in the binding site, the whole protein, NADPH and "crystallographic" water molecules W4.

\begin{tabular}{|c|c|c|c|c|c|c|c|c|c|}
\hline \multirow{2}{*}{$\begin{array}{l}\text { PTX } \\
\text { interactions }\end{array}$} & \multicolumn{3}{|c|}{$\begin{array}{c}\text { wt hDHFR-NADP-PTX } \\
\text { X ray+200 MIN }\end{array}$} & \multicolumn{3}{|c|}{$\begin{array}{c}\text { wt hDHFR-NADPH-PTX } \\
10 \mathrm{ps}^{+} 200 \mathrm{MIN}\end{array}$} & \multicolumn{3}{|c|}{$\begin{array}{c}\text { L22F hDHFR-NADPH-PTX } \\
30 \mathrm{ps}+200 \mathrm{MIN}\end{array}$} \\
\hline & Coulomb & VdW & Total & Coulomb & VdW & Total & Coulomb & VdW & Total \\
\hline Ile-7 & -2.8 & -0.4 & -3.2 & -2.7 & -0.5 & -3.2 & -1.3 & -0.1 & -1.4 \\
\hline Asp-21 & -0.7 & -0.7 & -1.3 & -0.3 & -0.2 & -0.6 & -0.6 & -0.1 & -0.8 \\
\hline Leu/Phe-22 & 1.2 & -2.4 & -1.1 & 0.8 & -1.2 & -0.4 & 0.4 & -1.8 & -1.3 \\
\hline Arg-28 & -3.9 & -0.1 & -4.0 & -4.9 & -0.1 & -5.0 & -3.4 & -0.2 & -3.6 \\
\hline Glu-30 & -66.5 & 4.2 & -62.3 & -65.7 & 4.1 & -61.6 & -67.6 & 4.4 & -63.3 \\
\hline Phe-31 & 0.0 & -1.9 & -1.9 & 0.3 & -1.9 & 1.6 & -0.4 & -2.5 & -2.8 \\
\hline Arg-32 & -2.8 & -0.1 & -2.9 & -5.4 & -0.1 & -5.5 & -4.6 & -0.1 & -4.6 \\
\hline Phe-34 & 2.1 & -2.7 & -0.7 & 2.0 & -2.4 & -0.4 & 2.2 & -2.4 & -0.2 \\
\hline Val-115 & -4.8 & -0.5 & -5.4 & -5.5 & -0.1 & -5.7 & -5.1 & -0.1 & -5.2 \\
\hline Protein & -71.7 & -15.2 & -86.9 & -88.2 & -13.2 & -101.4 & -91.9 & -13.2 & -105.1 \\
\hline NADPH & -27.1 & -2.7 & -29.8 & -33.3 & -2.6 & -35.9 & -31.6 & -2.0 & -33.6 \\
\hline W4 & -3.2 & -0.2 & -3.3 & -1.8 & 0.5 & -1.4 & -3.4 & 0.2 & -3.2 \\
\hline
\end{tabular}

Other individual amino acids contribute less then $2 \mathrm{kcal} / \mathrm{mol}$. The strong stabilization of PTX due to NADPH is $90 \%$ of an electrostatic character. We found that water does not interact strongly with PTX in ternary complexes. The data presented in Table 4 show also that the relative interaction energies do not change much after MD simulations or L22F mutation. A better fit of the PTX ligand, observed in the wt hDHFR-NADPH-PTX complex after 10 ps dynamics and 200 steps of minimization, results in a minor increase of the NADPH-PTX interaction energy from 30 $\mathrm{kcal} / \mathrm{mol}$ found in the X-ray structure to 36 $\mathrm{kcal} / \mathrm{mol}$ after dynamics.

Data presented in Table 4 and Table 5 indicate that there is no difference in the PTX-protein interaction between the wt and the L22F variant since the accuracy of the theoretical approach adopted here does not exceed $2-5 \mathrm{kcal} / \mathrm{mol}$. The experimental values of the inhibition constant of PTX are $K(\mathrm{i})=$ $0.0077 \mathrm{nM}$ and $0.659 \mathrm{nM}$ for these proteins, respectively [5]. Thus, the observed 86 -fold de- crease of PTX affinity towards L22F should perhaps be attributed to differences in the hydration mode of these proteins. Such effects were not studied here.

However, when the total PTX interaction energies with the protein and NADPH are considered, the cooperative effect of NADPH on the PTX binding is less clear. In the relaxed ternary complexes we have found values of -137 to $-139 \mathrm{kcal} / \mathrm{mol}$, while in the wt binary complex the value of the PTX-protein interaction energy is almost the same, i.e. -133 $\mathrm{kcal} / \mathrm{mol}$ (Table 5). One should note that nearly $10 \%$ of this rather large protein-PTX interaction energy is contributed by the Asp21 residue. This amino acid had no formal charge in our ternary models, but the formal charge of -1 was used in the binary models. The charged Asp21 residue in the binary systems was introduced in order to keep the total charge in all models the same. An additional PTX stabilization of $-5 \mathrm{kcal} / \mathrm{mol}$ comes from those water molecules (W2) which replaced $\mathrm{NADPH}$ in the model of the binary complex. 
Table 5. Piritrexim (PTX) intermolecular interaction energy (kcal/mol) calculated for $\mathrm{wt} \mathrm{hDHFR} \mathrm{and}$ E30A hDHFR binary complexes using the ESFF forcefield.

The calculated energy is partitioned between the selected amino acids in the binding site, whole protein, water molecules which replaced NADPH (W2) and "crystallographic" water (W4).

\begin{tabular}{lrrrrrr}
\hline \multirow{2}{*}{ PTX interactions } & \multicolumn{2}{c}{ wt hDHFR-PTX $10 \mathrm{ps}+500 \mathrm{MIN}$} & \multicolumn{3}{c}{ E30A hDHFR-PTX 3 ps+200 MIN } \\
\cline { 2 - 7 } & Coulomb & vdW & Total & Coulomb & VdW & Total \\
\hline Ile-7 & -2.2 & -0.8 & -3.0 & -2.7 & -0.8 & -3.5 \\
Asp-21 & -11.9 & -0.3 & -12.2 & -16.5 & -0.1 & -17.1 \\
Leu-22 & 0.9 & -1.1 & -0.2 & 1.0 & -2.2 & -1.2 \\
Arg-28 & -5.2 & -0.4 & -5.6 & -5.5 & -0.1 & -5.7 \\
Glu/Ala-30 & -67.6 & 3.5 & -64.1 & -1.1 & -0.3 & -1.9 \\
\hline Phe-31 & 0.1 & -2.7 & -2.6 & -0.1 & -2.3 & -2.5 \\
Arg-32 & -4.8 & -0.1 & -4.9 & -5.1 & -0.1 & -5.1 \\
Phe-34 & 2.4 & -2.3 & 0.1 & 1.6 & -2.2 & -0.6 \\
Arg-36 & -0.9 & 0.0 & -0.9 & -2.2 & 0.0 & -2.2 \\
Val-115 & -1.6 & -0.5 & -2.1 & -2.0 & -0.7 & -2.7 \\
Protein & -117.6 & -15.4 & -133.1 & -56.0 & -19.2 & -75.2 \\
W2 & -4.2 & -1.2 & -5.3 & -11.0 & -0.9 & -11.7 \\
W4 & 2.2 & 0.0 & 2.1 & -6.1 & 0.9 & -5.3 \\
\hline
\end{tabular}

The effect of cooperativity may result from either local, direct interaction or a global change in the protein structure leading to a better (or worse) fit of the ligand under study. In the case of the NADPH-hDHFR-PTX system, the direct NADPH-PTX interactions are strong and lead to positive cooperativity. On the other hand, this study also shows that the global structural changes induced by NADPH binding to hDHFR are substantial. The changes in PTX binding pocket geometry result in weaker protein-PTX interactions (negative cooperativity). Our data show that the positive (local) effect is slightly stronger than the negative (global) one.

The structural differences between the binary and wt ternary complexes are shown in Fig. 3. The fold of the protein is well conserved. The major changes induced by NADPH binding are localized to the vicinity of the cofactor. The PTX binding site is similar in all the structures compared. The position of helix A is maintained in all complexes. In the binary complex the shift in the Pro25-Pro26

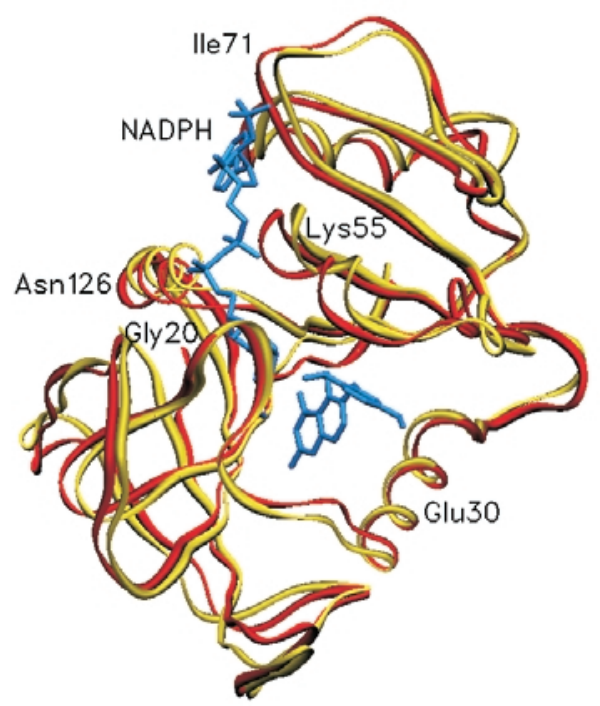

Figure 3. Overlap of a snapshot (10 ps) from the ESFF trajectory of the hDHFR-PTX binary complex (red) with the crystal structure of the hDHFR-NADPH-PTX ternary complex.

The protein backbone is yellow, the ligands are blue.

fragment of loop I results in closure of the pABA-Glu site: the distances between $\mathrm{C} \beta$ atoms of Pro26 and Pro61 are $7.2 \AA$ and $4.7 \AA$ 
for the crystal structure of the ternary complex and the wt binary complex, respectively (Fig. 1).

The lack of the NADPH coenzyme bound to DHFR triggered a shift of the helix B (residues 53-63) along its axis away from the PTX binding site. The maximum difference in $\mathrm{C} \alpha$ position between the crystal structure and the binary wt complex (2.6 $\AA$ ) is observed for Lys55 of helix B, the amino acid involved in the binding of the NADPH pyrophosphate. A similar motion of helix $\mathrm{C}$, equivalent to the hDHFR helix B, has been reported for bacterial DHFR [12, 33].

In the binary wt hDHFR model the helices $\mathrm{C}$ and $\mathrm{D}$, constituting the adenyl-ribose binding site, move from their positions observed in the crystal structure of the ternary complex [10], which results in a local opening of the cleft. Such structural trend indicates that the polar interactions of the pyrophosphate moiety of NADPH bridge the $\mathrm{C}$ and D helices. No direct coupling of this motion with the PTX site is observed. However, the relocation of helix $\mathrm{C}$ in the binary complex seems to be coupled with the motion of helix $\mathrm{B}$ discussed above.

Loop I (vicinity of residue 20, yellow in Fig. 1) is a part of the nicotinamide-ribose binding site. After NADPH removal this loop changes its position by about $1 \AA$ with respect to the ternary crystal structure and is positioned slightly closer to helix B (Lys55). Consequently, the part of the cleft in which the nicotinamide moiety of NADPH is bound in the ternary complexes is narrowed in the absence of the coenzyme. However, the binary complex of PTX with the E30A variant of hDHFR does not reveal such conformational changes. Its structure is very similar to the crystal structure of the ternary complex of PTX with wt DHFR.

Loop I is equivalent to the M20 loop of bacterial DHFR. The large flexibility of the M20 loop observed in crystals of ecDHFR has been linked with its role in catalytic activity [12]. More recently significant differences between the binary and ternary MTX complexes of pcDHFR have been detected [32]. It has been found that a movement of the loop region near Asn23 as large as $7 \AA$ is possible. A new 'flap-open' conformation of this loop in the binary complex was observed [32]. During our simulations of hDHFR loop I remained in the same conformation in all the systems studied so no effect of NADPH was observed. On the sub-nanosecond time scale of simulations no significant conformational changes were observed which could be linked to the closed, occluded or open conformations of the M20 loop discussed for the bacterial DHFR [12].

A detailed comparison of the binary and ternary complexes (Fig. 4) reveals a shift of the

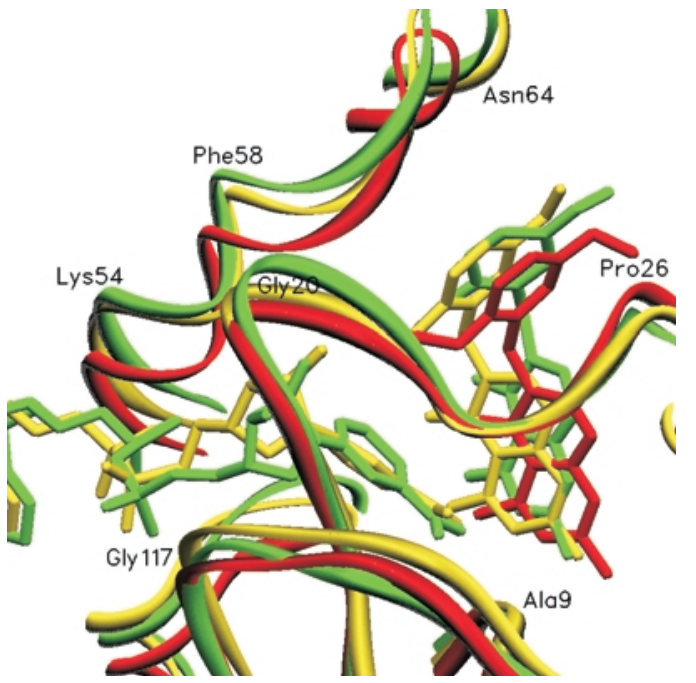

Figure 4. Structural changes induced by NADPH binding.

A superposition of the crystal wt ternary complex structure [10] (yellow), the L22F ternary complex (green) and the wt hDHFR-PTX binary complex (red).

inhibitor towards the Glu30 residue due to the lack of NADPH interactions. Energy calculations showed that the PTX-Glu30 interaction energy is indeed the highest in the binary complex (Tables 4 and 5). The conformation of PTX methylene bridge is not affected by NADPH and the torsion angles vary by less than $30^{\circ}$. Again, a rather large conformational freedom of methoxy groups of PTX is observed, similar to our previous study [11]. The main structural change found during our 
a

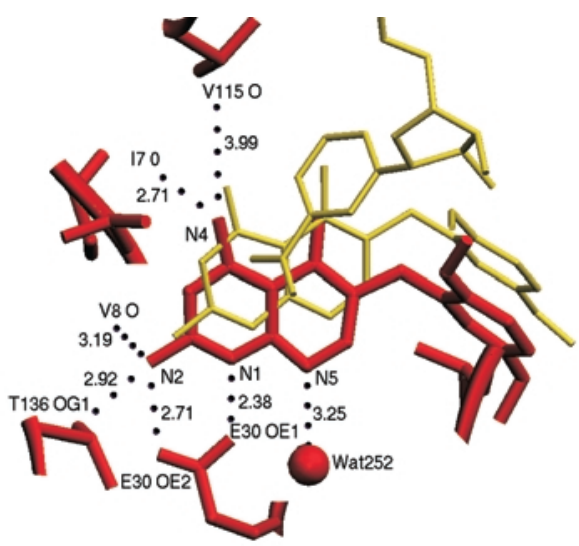

b

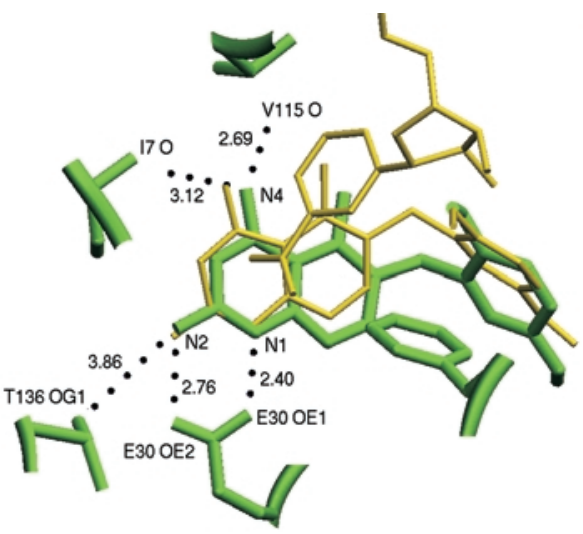

c

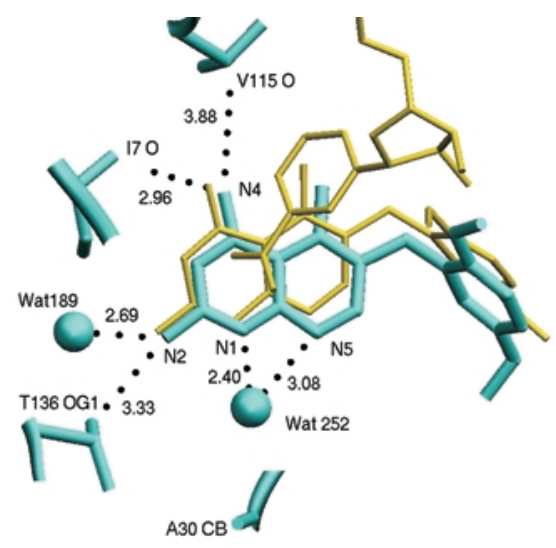

Figure 5. Superposition of the ternary hDHFR-NADPH-PTX complex (yellow) [10] with the wt hDHFR-PTX binary complex (red), the ternary complex of the L22F variant (30 ps MD run, green) and the binary complex of the E30A variant (blue).

a, The change of piritrexim (PTX) position towards Glu30 observed in the wt hDHFR-PTX binary complex; b, the Phe22 side chain causes a shift of the ligand dimethoxyphenyl moiety, while the pteridine ring orientation is preserved; c, the position of PTX is maintained, the Wat252 water molecule mimics the binding interactions of the missing Glu30. simulations is a partial collapse of the cofactor binding cleft in the absence of NADPH, which is clearly seen in Fig. 4 (helix B and loop I regions). It is worth noting that the L22F mutation does not affect PTX binding to Glu30.

\section{Details of PTX binding}

During the MD simulation, a water molecule remained docked in the PTX inhibitor binding cavity of binary complexes (Fig. 5a, 5c). This water molecule forms an additional $3.25 \AA$ hydrogen bond to the PTX N5 atom and another water molecule. A similar water molecule was also found in the active site in the crystal structure of the ternary complex [10].

In the ternary complex of the L22F variant of DHFR PTX orientation is similar to that found in the crystal structure of the wt DHFR ternary complex (Fig. 5b). The observed differences reflect the increased volume and rigidity of Phe22, when compared with the Leu22 residue of the wild type DHFR. The interactions with nicotinamide prevented the PTX from drifting towards Glu30. No direct interactions of water a molecule with the PTX N5 atom were detected.

\section{PTX binding in the E30A variant of hDHFR}

The positioning role of the residue equivalent to human DHFR Glu30 in bacterial DHFR has been discussed ([33] and references therein). Kinetic data have shown that Asp27 role in ecDHFR is perhaps related to substrate protonation [33]. On the other hand, one might expect that without a strong interaction with Glu30, the inhibitor would not be docked in hDHFR so effectively. To check the role of Glu30 in PTX binding, we have replaced the large, charged glutamic acid with a smaller, hydrophobic alanine. During MD simulations of the binary complex of the E30A variant, the position of piritrexim was maintained and remained close to the position observed in the crystal structure of the ternary complex. The water molecule found in the binding site is po- 
sitioned in the same space as the OE1 atom of the carboxylic group of the Glu30 side chain (Fig. 5c). This water molecule forms two polar interactions to $\mathrm{N} 1$ and N5 of the PTX pteridine ring, substituting for the missing Glu30 interactions. The polar interactions to Ile7, Thr 136 and Val115 are maintained in the binary complexes of wt DHFR and the E30A variant (Fig. 5). These results indicate that ligand/substrate positioning is achieved without any contribution of the conserved carboxylic group of the residue Glu30 or Asp27 (in human and bacterial DHFR, respectively). Therefore, the modeling gives additional arguments for the role of the conserved carboxylic group as a polarizing agent, which facilitates the buildup of a positive charge on the substrate's pteridine ring.

\section{CONCLUSIONS}

The ESFF forcefield gives reliable results for the geometry of hDHFR-PTX binary complexes, and therefore might be used as a routine approach to modeling studies of complexes with new ligands of interest. The distribution of amino acids on the Ramachandran plot among the three allowed sections is reasonably good. Special caution should be devoted to the treatment of the polypeptide omega angle $(\mathrm{C} \alpha-\mathrm{C}-\mathrm{N}-\mathrm{C} \alpha)$ which tends to loose its planarity during ESFF modeling.

The orientation of the NADPH ligand in the model complex is similar to that observed in the crystal structure. Despite some relaxation due to the nature of the forcefield potentials, all important ligand-protein interactions are preserved during the energy minimization. Qualitative trends in the hierarchy of the interaction energy of the PTX ligand were successfully reproduced. Using the ESFF forcefield the strongest interactions of PTX are predicted for the Glu30 $(-60 \mathrm{kcal} / \mathrm{mol})$ and NADPH ( $-33 \mathrm{kcal} / \mathrm{mol})$.

The main structural change observed in the binary hDHFR-PTX complexes after NADPH dissociation is the collapse of the NADPH binding cleft in the vicinity of helix B and loop I region. The PTX-protein interactions are stronger in binary complexes than those in hDHFR-NADPH-PTX ternary complexes. The direct stabilizing NADPH-PTX interactions, however, compensate for the global effects of protein relaxation. Thus a positive cooperative effect of NADPH on PTX binding is expected in the hDHFR protein.

Our MD simulations suggest that loop I (Fig. 1), important for substrate binding, is rather rigid in all forms of the human enzyme studied. This observation is in contrast to the large flexibility of the equivalent loop M20 observed in ecDHFR [12].

Theoretical data indicate that PTX in the E30A variant of the binary complex of hDHFR is well bound. The lack of Glu30-PTX interactions substantially reduces the interaction energy (by about $60 \mathrm{kcal} / \mathrm{mol}$ ) but the ligand is still kept tightly in the model complex. This indicates that the polar residues Glu30 (or Asp27 in ecDHFR) are not critical for substrate binding, and their role is rather to facilitate charge redistribution on the folate than to keep the ligand in the pocket. This is in keeping with the kinetic data observed for a series of F31X variants that showed the $K(\mathrm{i})$ for MTX binding changed to $265 \mathrm{pM}$ for F31A compared with $3.4 \mathrm{pM}$ for the wild type hDHFR [4].

Future prospects for modeling using ESFF are good. Every month brings new, exciting DHFR structures from such medically important species as Mycobacterium tuberculosis [34], Pneumocystis carinii [32] or Lactobacillus casei [35], which opens new possibilities for theoretical studies of the dynamics of these enzymes and rational drug design using universal force fields.

Computational results were obtained using software from Molecular Simulations Inc. the dynamics calculations were done with the Discover3 module. Figures 1 and 2 were 
printed out from the InsightII molecular modeling system. Other figures were prepared with program SETOR [36].

\section{R E F E R E N C E S}

1. Blakley, R.L. (1995) Advances in Enzymology and Related Areas of Molecular Biology; vol. 70, pp. 23-102, John Wiley \& Sons, Inc.

2. Takimoto, C.H. (1996) New antifolates: Pharmacology and clinical applications. Oncologist 1, 68-81.

3. Patel, M., Sleep, S.E., Lewis, W.S., Spencer, H.T., Mareya, S.M., Sorrentino, B.P. \& Blakley, R.L. (1997) Comparison of the protection of cells from antifolates by transduced human dihydrofolate reductase mutants. Hum. Gene Ther. 8, 2069-2077.

4. Chunduru, S. K., Cody, V., Luft, J.R., Pangborn, W., Appleman, J.R. \& Blakley, R.L. (1994) Methotrexate-resistant variants of human dihydrofolate reductase. Effects of Phe31 substitutions. J. Biol. Chem. 269, 9547-9555.

5. Lewis, W.S., Cody, V., Galitsky, N., Luft, J.R., Pangborn, W., Chunduru, S.K., Spencer, H.T., Appleman, J.R. \& Blakley, R.L. (1995) Methotrexate-resistant variants of human dihydrofolate reductase with substitutions of leucine 22. Kinetics, crystallography, and potential as selectable markers. J. Biol. Chem. 270, 5057-5064.

6. Grivsky, E.M., Lee, S., Sigel, C.W. \& Nichol, C.A. (1980) Synthesis and antitumor activity of 2,4-diamino-6-(2,5-dimethoxybenzyl)-5-methylpyrido[2,3-d]pyrimidine. J. Med. Chem. 23, 327-329.

7. Khorsand, M., Lange, J., Feun, L., Clendeninn, N.J., Collier, M. \& Wilding, G. (1997) Phase II trial of oral piritrexim in advanced, previously treated transitional cell cancer of bladder. Invest. New Drugs 15, 157-163.
8. Graffner-Nordberg, M., Kolmodin, K., Aqvist, J., Queener, S.F. \& Hallberg, A. (2001) Design, synthesis, computational prediction, and biological evaluation of ester soft drugs as inhibitors of dihydrofolate reductase from Pneumocystis carinii. J. Med. Chem. 44, 2391-2402.

9. Cinquina, C.C., Grogan, E., Sun, R., Lin, S.F., Beardsley, G.P. \& Miller, G. (2000) Dihydrofolate reductase from Kaposi's sarcoma-associated herpesvirus. Virology 268, 201-217.

10. Cody, V., Wojtczak, A., Kalman, T.I., Freisheim, J.H. \& Blakley, R.L. (1993) in: Chemistry and Biology of Pteridines and Folates (Ayling, J.E., Nair, M.G. \& Baugh, C.M., eds.) pp. 481-486, Plenum Press.

11. Nowak, W., Wojtczak, A. \& Cody, V. (1999) Computer modeling of human dihydrofolate reductase ternary complex with NADPH and piritreim (PTX) inhibitor. Internet J. Chem. 12, www.ijc.com/articles/1999v2/50/.

12. Sawaya, M.R. \& Kraut, J. (1997) Loop and subdomain movements in mechanism of Escherichia coli dihydrofolate reductase: Crystallographic evidence. Biochemistry 36, 586-603.

13. Miller, P.G. \& Benkovic, S.J. (1998) Stretching exercises - flexibility in dihydrofolate reductase catalysis. Chem. Biol. 5, R105-R113.

14. Osborne, M.J., Schnell, J., Benkovic, S.J., Dyson, H.J. \& Wright, P.E. (2001) Backbone dynamics in dihydrofolate reductase complexes: Role of flexibility in the catalytic mechanism. Biochemistry 40, 9846-9859.

15. Johnson, J.M., Meiering, E.M., Wright, J.E., Pardo, J., Rosowsky, A. \& Wagner, G. (1997) NMR solution structure of the antitumor compound PT523 and NADPH in the ternary complex with human dihydrofolate reductase. Biochemistry 36, 4399-4411.

16. Cody, V., Galitsky, N., Luft, J.R., Pangborn, W., Rosowsky, A. \& Blakley, R.L. (1997) Comparison of two independent crystal structures of human dihydrofolate reductase ternary 
complexes reduced with nicotinamide adenine dinucleotide phosphate and the very tight-binding inhibitor PT523. Biochemistry 36, 13897-13903.

17. Pan, H., Lee, J.C. \& Hilser, V.J. (2000) Binding sites in Escherichia coli dihydrofolate reductase communicate by modulating the conformational ensemble. Proc. Natl. Acad. Sci. U.S.A. 97, 12020-12025.

18. Bystroff, C. \& Kraut, J. (1991) Crystal structure of unliganded Escherichia coli dihydrofolate reductase. Ligand-induced conformational changes and cooperativity in binding. Biochemistry 30, 2227-2239.

19. Carugo, O. \& Argos, P. (1997) NADP-dependent enzymes. I: Conserved stereochemistry of cofactor binding. Proteins 28,10-28.

20. Whitlow, M., Howard, A.J., Stewart, D., Hardman, K.D, Chan, J.H., Baccanari, D.P., Tansik, R.L., Hong, J.S. \& Kuyper, L.F. (2001) X-Ray crystal structures of Candida albicans dihydrofolate reductase: High resolution ternary complexes in which the dihydronicotinamide moiety of NADPH is displaced by an inhibitor. J. Med. Chem. 44, 2928-2932.

21. Verma, C.S., Caves, L.D.S., Hubbard, R.E. \& Roberts, G.C.K. (1997). Domain motions in dihydrofolate reductase: A molecular dynamics study. J. Mol. Biol. 266, 776-796.

22. Graffner-Nordberg, M., Marelius, J., Ohlsson, S., Persson, A., Swedberg, G., Andersson, P., Andersson, S.E., Aqvist, J. \& Hallberg, A. (2000) Computational predictions of binding affinities to dihydrofolate reductase: Synthesis and biological evaluation of methotrexate analogues. J. Med. Chem. 43, 3852-3861.

23. Pitts, C., Bowen, D. \& Southerland, W.M. (2000) Interaction energy analyses of folate analog binding to human dihydrofolate reductase: Contribution of the antifolate substructural regions to complex stability. Drug Metabol. Drug Interact. 16, 99-121.
24. Cummins, P.L. \& Gready, J.E. (2001) Energetically most likely substrate and active-site protonation sites and pathways in the catalytic mechanism of dihydrofolate reductase. $J$. Am. Chem. Soc. 123, 3418-3428.

25. Verkhivker, G.M., Rejto, P.A., Bouzida, D., Arthurs, S., Colson, A.B., Freer, S.T., Gehlhaar, D.K., Larson, V., Luty, B.A., Marrone, T. \& Rose, P.W. (2001) Navigating ligand-protein binding free energy landscapes: Universality and diversity of protein folding and molecular recognition mechanisms. Chem. Phys. Lett. 336, 495-503.

26. Sham, Y.Y., Ma, B., Tsai, C.J. \& Nussinov, R. (2001) Molecular dynamics simulation of Escherichia coli dihydrofolate reductase and its protein fragments: Relative stabilities in experiment and simulations. Protein Sci. 10, $135-348$.

27. Barlow, S., Rohl, A.L., Shi, S.G., Freeman, C.M. \& O'Hare, D. (1996) Molecular mechanics study of oligomeric models for poly(ferrocenylsilanes) using the Extensible Systematic Forcefield (ESFF). J. Am. Chem. Soc. 118, 7578-7592.

28. Forcefield-Based Simulations' (1997) Molecular Simulations Inc., San Diego.

29. Andersen, H.C. (1983) RATTLE: A velocity version of the SHAKE algorithm for molecular dynamics. J. Comp. Physics 52, 24-34.

30.Laskowski, R.A., MacArthur, M.W., Moss, D.S. \& Thornton, J.M. (1993) PROCHECK: A program to check the stereochemical quality of protein structures. J. Appl. Crystallogr. 26, 283-291.

31. Brunger, A.T. (1992) X-PLOR Version 3.1: A System for X-Ray Crystallography and NMR, Yale University Press, New Haven.

32. Cody, V., Galitsky, N., Rak, D., Luft, J.R., Pangborn, W. \& Queener, S.F. (1999) Ligandinduced conformational changes in the crystal structures of Pneumocystis carinii dihydro- 
folate reductase complexes with folate and $\mathrm{NADP}^{+}$. Biochemistry 38, 4303-4312.

33. Howell, E.E., Villafranca, J.E., Warren, M.S., Oatley, S.J. \& Kraut, J. (1986) Functional role of aspartic acid-27 in dihydrofolate aeductase revealed by mutagenesis. Science 231, 1123-1128.

34.Li, R., Sirawaraporn, R., Chitnumsub, P., Sirawaraporn, W., Wooden, J., Athappilly, F., Turley, S. \& Hol, W.G. (2000) Three-dimensional structure of $M$. tuberculosis dihydrofolate reductase reveals opportunities for the design of novel tuberculosis drugs. J. Mol. Biol. 295, 307-323.
35. Gargaro, A.R., Soteriou, A., Frenkiel, T.A., Bauer, C.J., Birdsall, B., Polshakov, V.I., Barsukov, I.L., Roberts, G.C.K. \& Feeney, J. (1998) The solution structure of the complex of Lactobacillus casei dihydrofolate reductase with methotrexate. J. Mol. Biol. 277, 119-134.

36. Evans, S.V. (1993) SETOR: Hardware lighted three-dimensional solid model representations of macromolecules. J. Mol. Graphics 11, 134-138. 